\title{
Mathematical Simulation for Integrated Linear Fresnel Spectrometer Chip
}

\author{
Yeonjoon Park ${ }^{\mathrm{a}}$, Hargsoon Yoon ${ }^{\mathrm{b}}$, Uhn Lee $^{\mathrm{c}}$, Glen C King ${ }^{\mathrm{d}}$, Sang Choi ${ }^{\mathrm{d}}$ \\ ${ }^{a}$ National Institute of Aerospace, Hampton, VA 23666 \\ ${ }^{\mathrm{b}}$ Norfolk State University, Norfolk, VA 23504 \\ ${ }^{\mathrm{C}}$ Gacheon School of Medicine, Incheon, South Korea \\ ${ }^{\mathrm{d}}$ NASA Langley Research Center, Hampton, VA 23681
}

\begin{abstract}
A miniaturized solid-state optical spectrometer chip was designed with a linear gradient-gap Fresnel grating which was mounted perpendicularly to a sensor array surface and simulated for its performance and functionality. Unlike common spectrometers which are based on Fraunhoffer diffraction with a regular periodic line grating, the new linear gradient grating Fresnel spectrometer chip can be miniaturized to a much smaller form-factor into the Fresnel regime exceeding the limit of conventional spectrometers. This mathematical calculation shows that building a tiny motionless multi-pixel microspectrometer chip which is smaller than $1 \mathrm{~mm}^{3}$ of optical path volume is possible. The new Fresnel spectrometer chip is proportional to the energy scale (hc/ $\lambda$ ), while the conventional spectrometers are proportional to the wavelength scale $(\lambda)$. We report the theoretical optical working principle and new data collection algorithm of the new Fresnel spectrometer to build a compact integrated optical chip.
\end{abstract}

KEYWORDS: Micro Spectrometer Chip Design, Fresnel Diffraction, Linear Gradient Grating

\section{INTRODUCTION}

Recently, a circular Fresnel spectrometer with multiple zone plate rings is demonstrated with very small optical path volume below one cubic millimeter. ${ }^{1}$ However, a circular Fresnel spectrometer requires a certain number of mechanical parts to move the grating and aperture in order to guide the focal point of signal onto a sensor plane. Such mechanisms in a circular Fresnel spectrometer are bulky and unstable in vibration. To alleviate the problems associated with the complex mechanisms in a circular Fresnel spectrometer, we came up with an improved Fresnel spectrometer design which is suitable for a compact solid-state optical chip without moving parts and its theoretical performance using linear gradient Fresnel grating. 
A linear gradient Fresnel grating ${ }^{2,3,4}$ can be designed with the following equations.

The wavelength dependent focal distance $(f(\lambda))$ of a Fresnel grating is given by, $f(\lambda)=\frac{r^{2}}{\lambda \cdot l}$ where $r$ is the maximum distance of the grating line (which is similar to the radius of the zone plate), and $l$ is the total number of lines. The maximum distance of the grating is measured from the center of the grating in the case of circular and symmetric linear grating otherwise it is measured from the starting edge of the half linear gradient grating shown in Figure 1. In order to make photons of wavelength $\lambda$ to be situated at the focal distance $f(\lambda)$, the transparent and opaque lines have to be arranged between the distance $r(n+1, \lambda)$ and $r(n+2, \lambda)$ on the grating plate.

Here, the distance of $n_{\text {th }}$ line/ring is given by $r(n, \lambda)=n^{1 / 2} \cdot \sqrt{\lambda \cdot f(\lambda)}$

The square root dependence of the $r(n, \lambda)$ makes a gradient width and gradient gap distance of the Fresnel grating.

(a) Full circular

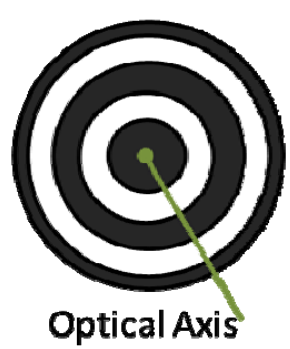

(b) Full linear

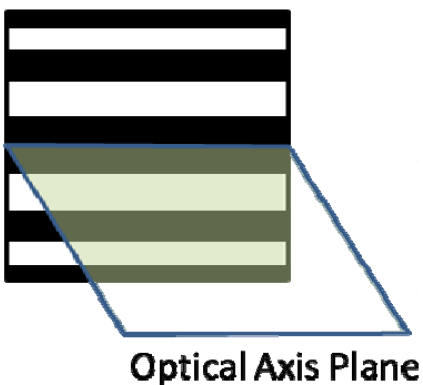

(c) Half circular

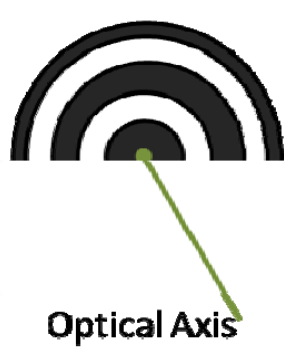

Optical Axis (d) Half linear

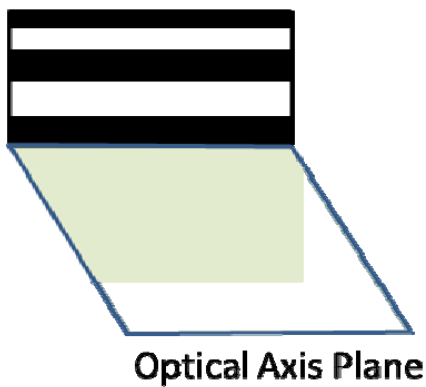

(e) Cross-section view of transmission type Fresnel grating

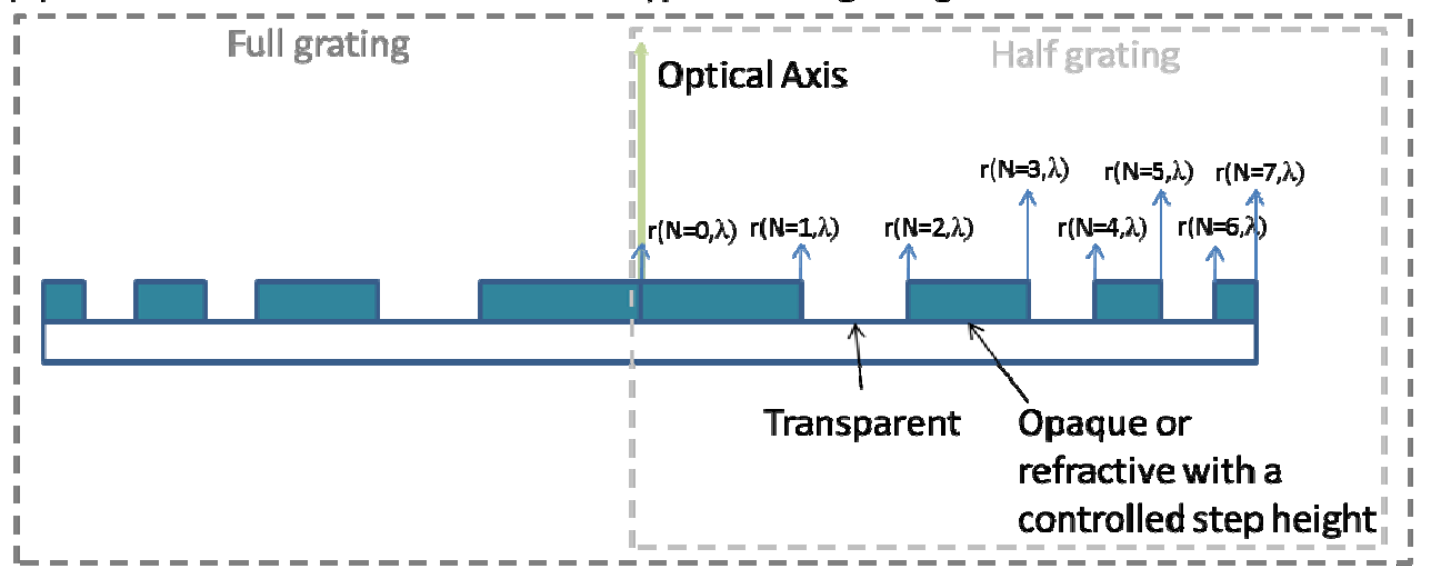

Figure 1: Various Fresnel gratings, (a) full circular (zone plate lens), (b) full linear gradient, (c) half circular, and (d) half linear gradient. (e) The definition of grating line/ring position on a transparent plate. 
Among the configurations in Figure 1, the optical axis plane of the half linear Fresnel grating can be occupied with a linear imaging sensor array because a commercial imaging sensor has an array of sensor lines in an active sensing area. The half linear one is also preferred because the typical imaging sensor allows a light entrance from space above the surface of the chip. The fabrication of such a linear gradient grating can be achieved using e-beam lithography, focused ion beam etching, or conventional UV photo lithography and wet etching. ${ }^{5}$

\section{MATHEMATICAL SIMULATION FOR LINEAR FRESNEL GRATING}

Figure 2 shows the comparison of two spectrometers, the conventional Fraunhofer spectrometer with a periodic regular line grating, and the integrated linear Fresnel spectrometer with a width-varying gradient line grating. While the conventional Fraunhofer spectrometer only works for a long optical path distance

$z \gg \frac{a^{2}}{\lambda}$ where $a$ is the size of grating aperture, the Fresnel spectrometer works at a very short optical path distance $z \ll \frac{a^{2}}{\lambda}$. Therefore, the linear Fresnel spectrometer chip can be fabricated in a very small chippackaging such that the height of the gradient linear grating is below $1 \mathrm{~mm}$ and the chip size is only a few millimeters while keeping the optical path volume below $1 \mathrm{~mm}^{3}$.

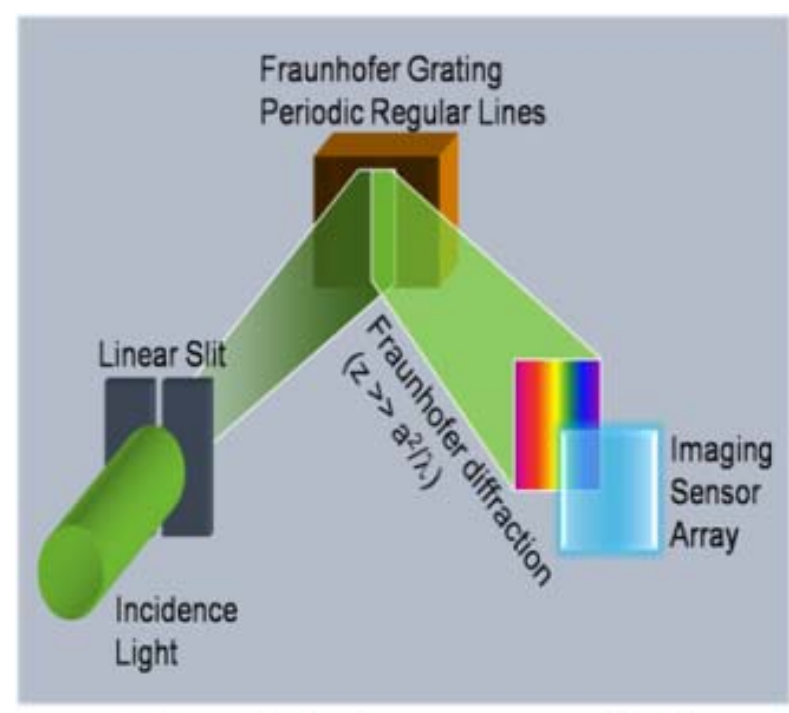

(1) Fraunhofer Spectrometer Mode

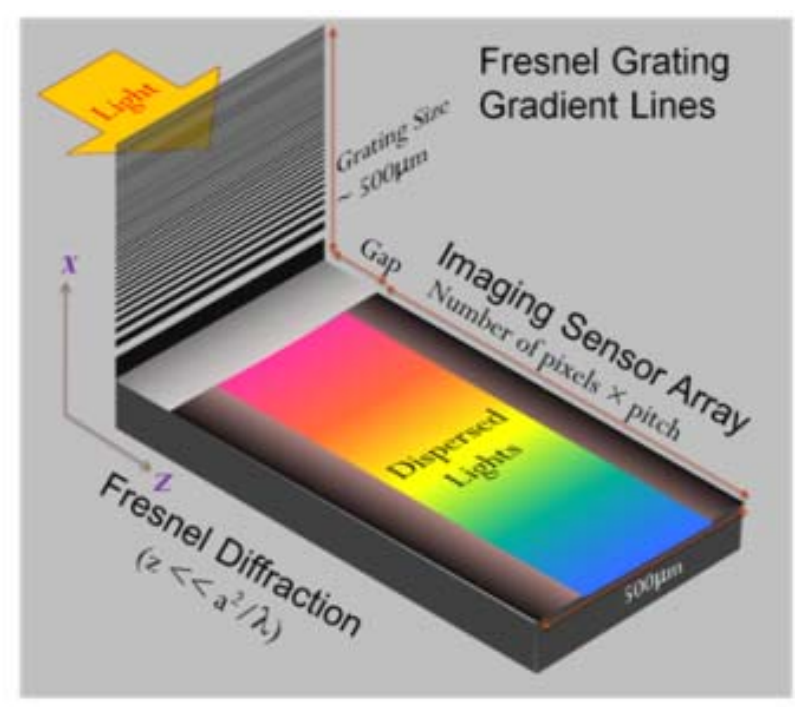

(2) Linear Fresnel Spectrometer Mode

Figure 2: Configuration of (1) the conventional Fraunhofer spectrometer and (2) linear Fresnel spectrometer.

The 90 degree vertical mount of the linear gradient grating on one end of the active sensing area allows photons to spread over the imaging sensor surface according to a proper spectral dispersion as shown in Figure 2-(2). In Figure 2, the optical distance from the bottom of the linear gradient grating to a line on 
the active sensing area is labeled as $\mathbf{Z}$, and the vertical distance from the surface of the sensing area is labeled as $\boldsymbol{x}$. These variables $\boldsymbol{z}$ and $\boldsymbol{x}$ will be used in the following mathematical calculations.

The light intensity per unit length, $I(z, \lambda, x)$ on the imaging sensor array surface is given by the following equations.

$u(n, z, \lambda, x)=$ Electric field per unit length by $(n+1) t h$ transparent line

$$
u(n, z, \lambda, x)=c(z, \lambda) \cdot e^{-i k(\lambda) \cdot z} \cdot\left[\int_{r(n+1, \lambda)}^{r(n+2, \lambda)} e^{-\left[\left(\frac{i \cdot k(\lambda)}{2 z}\right)(\xi-x)^{2}\right]} d \xi\right]
$$

where $c(z, \lambda)$ is the amplitude coefficient given by the incoming light intensity. ${ }^{6}$

$U(z, \lambda, x)=$ Total electric field per unit length from all transparent lines, $n=0,2,4,6 \ldots n_{f}-1$

$$
U(z, \lambda, x)=\sum_{m=0}^{\frac{n_{f}}{2}-1} u(2 m, z, \lambda, x)
$$

$I(z, \lambda, x)=$ The light intensity per unit length from the sum of all the electric field.

$$
I(z, \lambda, x)=|U(z, \lambda, x)|^{2}
$$

The light intensity $I(z, \lambda, x)$ shows the spatial distributions of photons of wavelength $\lambda$ on $\boldsymbol{z}$ and $\boldsymbol{x}$ plane which is the vertical cross-section of the imaging sensor's active area. Next Figure 3 shows the 3D plot of photon intensity on $\boldsymbol{z}$ and $\boldsymbol{x}$ plane. This optical simulation calculation is made with 500 micrometer $\mathrm{x} 500$ micrometer grating with the light spreading over a few millimeters' length ( $2 \mathrm{~mm}<\mathbf{z}<4 \mathrm{~mm}$ ) on the sensor surface. 


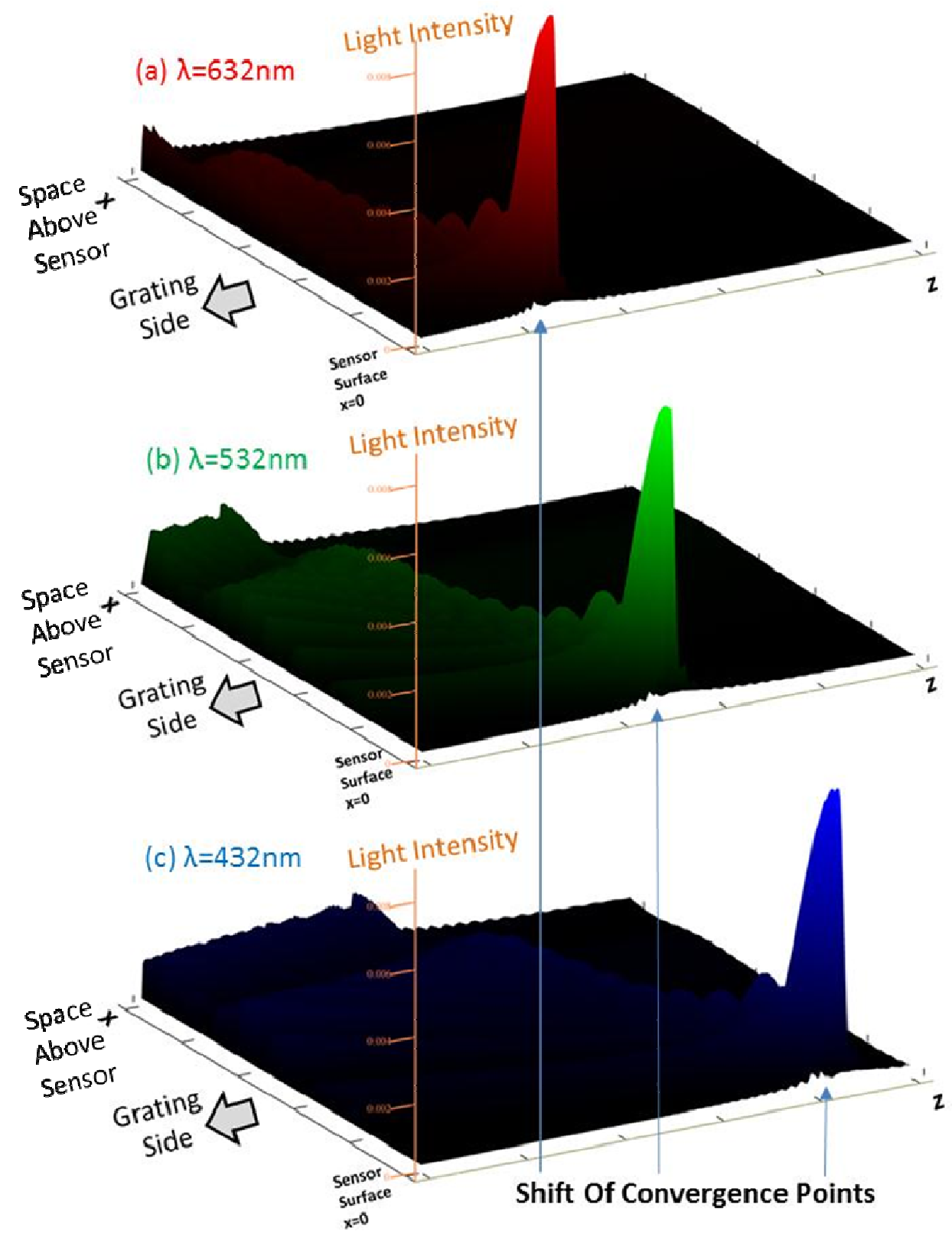

Figure 3: Light intensity distribution on $\mathbf{z}-\mathbf{x}$ plane $(\mathbf{x}>0$ is above the sensor surface. $\mathbf{z}$ is plotted near the focal points).

In Figure 3, $\mathbf{x}=0$ means the sensor surface and $\mathbf{x}>0$ is the empty space above the sensor. The simulation result shown in Figure 3 is made with $\mathbf{z}$ near the focal point of the grating. The linear gradient Fresnel grating focuses the light into a different convergence point which is determined by the wavelength of transmitting light. Note that the shift of the peaks on $\mathbf{x}=0$ is determined by the wavelength. The results are calculated with three wavelengths, $632 \mathrm{~nm}$ for red, $532 \mathrm{~nm}$ for green, and $432 \mathrm{~nm}$ for blue. This kind of 
Fresnel spectral dispersion is a founding basis to build a tiny spectrometer. It is fundamentally different from the underlying principle of Fraunhofer diffraction.

\section{NEW ALGORITHM TO OBTAIN WAVELENGTH FROM FRESNEL DISPERSION}

The simulation with a sinusoidal light distribution on the sensor pixels shows a clear difference between the conventional Fraunhofer spectrometer and the new Fresnel spectrometer such that while the Fraunhofer spectrometer's sensitivity is linear in the wavelength scale but reciprocal in the energy scale, the Fresnel spectrometer's sensitivity is linear in the energy scale but reciprocal in the wavelength scale. This difference is shown in Figure 4. This difference comes from the fact that the angular dispersion of Fraunhofer spectrometer is proportional to the wavelength $(\lambda)$, the focal line dispersion of Fresnel spectrometer is proportional to the 1 over wavelength, $1 / \lambda$ and the photon's energy $(E)$ is given by $E=h c / \lambda$ where $\mathrm{h}$ is a Planck's constant and $\mathrm{c}$ is the speed of light.

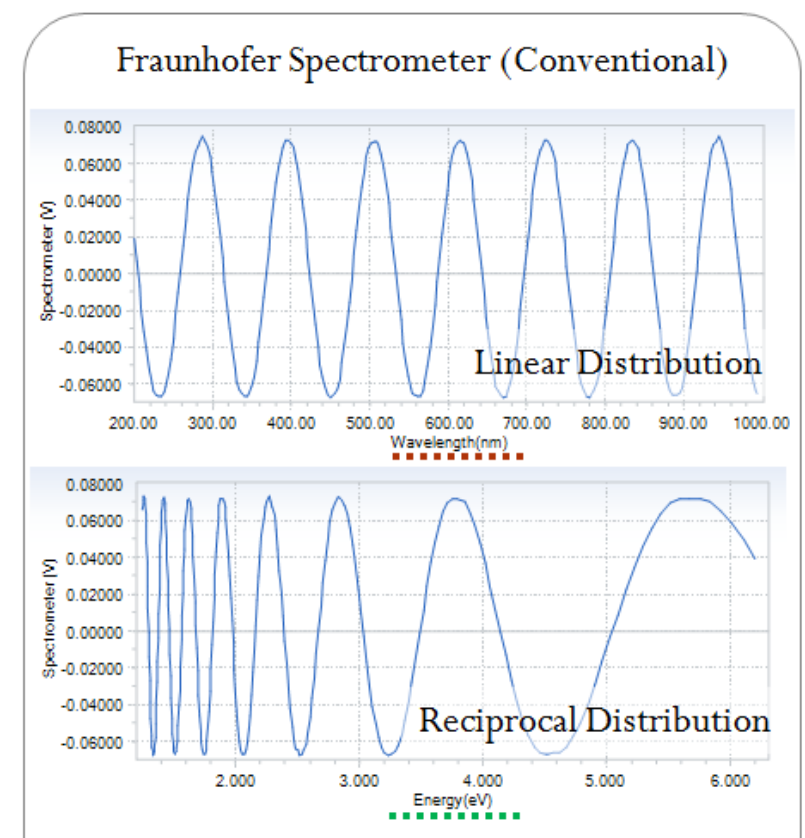

Fraunhofer spectrometer's sampling is linear to the wavelength scale $(\lambda=\mathrm{hc} / \mathrm{E})$.

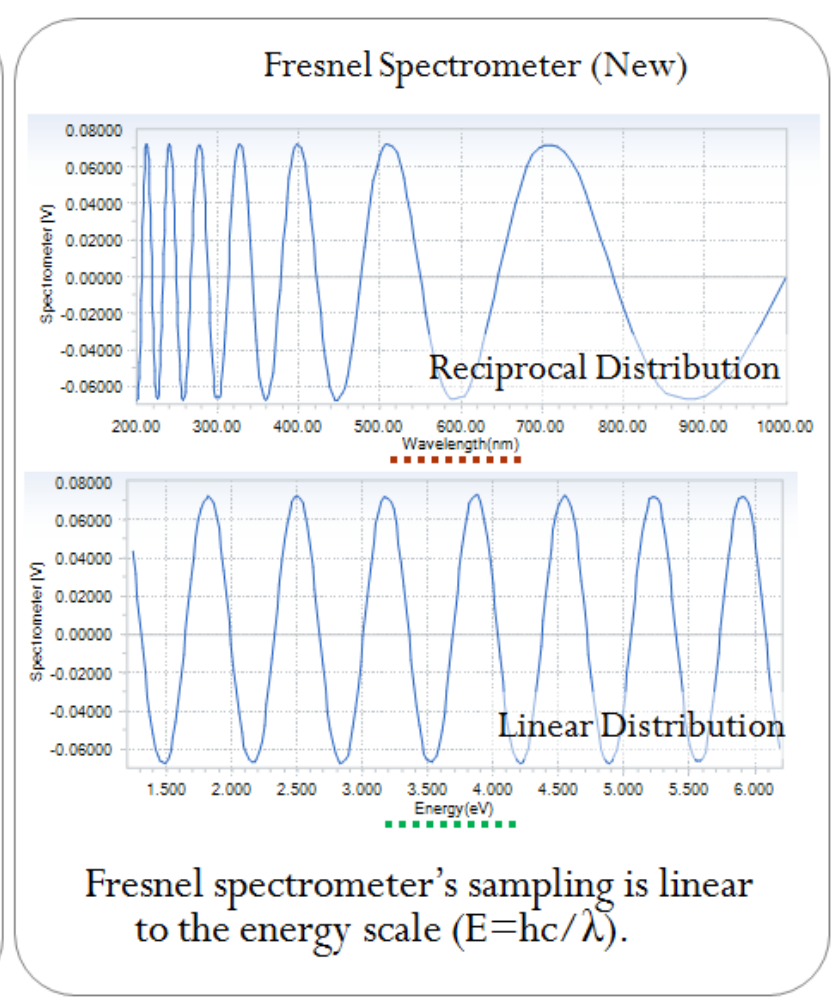

Fresnel spectrometer's sampling is linear to the energy scale $(\mathrm{E}=\mathrm{hc} / \lambda)$.

Figure 4: Sensor pixel distribution of Fraunhofer spectrometer and Fresnel spectrometer.

Therefore, a general data acquisition software for Fresnel spectrometer with multi-pixel sensor must interpret the spectrum signal distribution on pixels differently from the conventional Fraunhofer spectrometer. Here, we describe the details of this new algorithm. 
Let the imaging sensor has from 0 to $\mathrm{N}-1$ pixels, so totally $\mathrm{N}$ pixels. The optical distance $\mathrm{Z}$ is determined by

Optical distance $Z=$ gap distance between Fresnel grating and the $0^{\text {th }}$ pixel $+($ Pixel number $\times$ Pixel Pitch) where Pixel number, $\mathrm{n}$ is between 0 and N-1 and the Pixel Pitch is a sensor chip's constant.

Or, the offset distance from the edge to the center of the $0^{\text {th }}$ pixel can be included for an improved accuracy so that

Optical distance $Z=$ gap distance between Fresnel grating and the edge of the $0^{\text {th }}$ pixel + Pixel Pitch $/ 2+$ (Pixel number $\times$ Pixel Pitch).

Then, the wavelength of the photon on the $\mathrm{n}^{\text {th }}$ pixel (Pixel number $=\mathrm{n}$ ) with the Optical distance $\mathrm{Z}$ is determined by,

Wavelength $=K^{2} / Z$, where $K$ is a Fresnel Grating Size Constant such that

$$
K=\frac{R}{\sqrt{L}}
$$

$\mathrm{L}$ is the number of gradient rings (circular grating) or gradient lines (linear grating).

$\mathrm{R}$ is the radius of circular grating or the height of half linear grating.

The simplest scheme uses the Fresnel spectrometer's linearity in Energy scale so that the conversion to Energy is straight forward.

$$
\text { Energy }=\frac{h c L Z}{R^{2}}=\frac{h c L}{R^{2}}(\text { gap }+ \text { PixelNumber } * \text { PixelPitch })
$$

Or, if we include the distance from the edge of the pixel to the center for the pixel,

$$
\text { Energy }=\frac{h c L Z}{R^{2}}=\frac{h c L}{R^{2}}\left(\text { gap }+\frac{\text { PixelPitch }}{2}+\text { PixelNumber } * \text { PixelPitch }\right)
$$

Then, the wavelength is determined by

$$
\text { Wavelength }=\frac{h c}{\text { Energy }}
$$

\section{CONCLUSION}

The design and optical performance of a linear Fresnel spectrometer chip were demonstrated. The mathematical simulation with Fresnel diffraction was made for a size of $0.5 \mathrm{~mm} \times 0.5 \mathrm{~mm} \times 4 \mathrm{~mm}=1 \mathrm{~mm}^{3}$ volume of the optical path from the grating to the imaging pixels on the sensing area. This simulation 
result shows that a very compact and tiny spectrometer chip can be constructed using a linear gradient Fresnel grating which is vertically mounted on a conventional imaging sensor array chip. ${ }^{7}$ The newly developed multi-pixel data acquisition algorithm is very different from those of the conventional Fraunhofer spectrometers because Fresnel diffraction dispersion is proportional to the energy $(\mathrm{E}=\mathrm{hc} / \lambda)$.

\section{ACKNOWLEDGEMENT}

This research was supported by Space Act Agreement (SAA \#15546) between NASA Langley Research Center, Hampton, VA, USA and Gacheon University of Medicine and Science, Incheon, South Korea under KOSEF program of Korean Ministry of Education, Science and Technology (MEST).

\section{REFERENCES}

\footnotetext{
${ }^{1}$ Y. Park, L. Koch, K. D Song, S. Park, G. King, and S. Choi, Journal of Optics A: Pure and Applied Optics 10 (2008) 095301, doi:10.1088/1464-4258/10/9/095301

${ }^{2}$ F. Montiel and M. Nevi ere, J. Opt. Soc. Am. A/Vol. 12, No. 12/December 1995, p. 2672, DOI:10.1364/JOSAA.12.002672

${ }^{3}$ B. X. Yang, Nuclear Instruments and Methods in Physics Research A328 (1993) p. 578-587

${ }^{4}$ A. Sammar and J.-M. Andr6, J. Opt. Soc. Am. A/Vol. 10, No. 11/November 1993 p. 2324

${ }^{5}$ C. Davida, B. No hammera, E. Zieglerb, Microelectronic Engineering 61-62 (2002) 987-992

${ }^{6}$ Ghatak A and Thyagarajan K 1989 Optical Electronics, (New York: Cambridge University Press), ISBN-10: 0521314089

${ }^{7}$ Y. Park, S. Choi, NASA Invention Disclosures, LAR-17947, LAR-17946, Patent Pending
} 